\title{
A Utilização da Modelagem Computacional Semiquantitativa no Estudo do Sistema Mola-Massa
}

(The Use of Semiquantitative Computer Modelling in the Study of the Spring-Mass System)

\author{
Giuseppi Camiletti, e Laércio Ferracioli ${ }^{\dagger}$ \\ Departamento de Física, \\ Laboratório de Tecnologias Interativas Aplicadas à Modelagem Cognitiva \\ Universidade Federal do Espírito Santo \\ Campus de Goiabeiras, 29.060-900 Vitória, ES - Brasil
}

Recebido em 3 de abril, 2002. Aceito em 3 de junho, 2002.

\begin{abstract}
$\mathrm{O}$ artigo apresenta resultados da investigação sobre a integração de ambientes de modelagem computacional ao aprendizado exploratório de Física. Os resultados aqui apresentados são relativos ao estudo da interação e desempenho de alunos de ensino superior durante a utilização do ambiente de modelagem computacional semiquantitativo WLinkIt em uma atividade de conteúdo específico em Física: o sistema mola-massa. Os resultados mostram que os estudantes apresentaram habilidades para desenvolver um modelo sobre a situação proposta e relacionar o comportamento apresentado pelo modelo com o esperado por eles, alterar o modelo e explicar o comportamento apresentado pelas variáveis. Os resultados mostram também que as dificuldades apresentadas foram relacionadas à delimitação do sistema a ser estudado, ao entendimento da influência de uma variável sobre a outra, ao entendimento de quem é o agente causal do sistema, ao entendimento da função de uma ligação entre duas variáveis e ao entendimento dos conceitos envolvidos. Assim, o mapeamento desses aspectos é fundamental para o delineamento de pesquisas futuras no sentido de promover, na prática, a integração de Ambientes de Modelagem Computacional Semiquantitativos na sala de aula, mais especificamente para o estudo de tópicos de Física.
\end{abstract}

This article presents results about an investigation on the integration of computer modelling environment to the exploratory learning in Physics. The study is relative to the interaction and performance of university students acting during the use of the semiquantitative computer modelling environment WLinklt in an activity of specific content in Physics: the spring-mass system. The results show that the students presented abilities to develop a model about the proposed system and to relate and make comparison among the results of the model simulation with their previous expectation to modify the model and to explain the behaviour presented by the variables after a simulation. Regarding the difficulties these were related to the delimitation of the system to be studied, the inability of understanding the influence of a variable on the other and what is the causal agent of the system and to the understanding of the function of a connection among two variables and the involved concepts. Therefore, these results are fundamental for the design and development of future researches aiming at promoting, in the practice, the integration of semiquantitative computer modelling environments in the classroom, more specifically for the study of topics of Physics.

\section{Introdução}

A questão da utilização de novas tecnologias no contexto educacional tem sido discutida tanto em nível nacional quanto internacional. O governo federal destinou recursos em nível nacional para as Escolas de Ensino Médio para a aquisição de computadores, sem contudo definir claramente as diretrizes para a aquisição de softwares e ambientes computacionais educacionais.

Esse fato gera a necessidade do desenvolvimento de pesquisa sobre como promover, na prática, a integração dos recursos da tecnologia da informática com a sala de aula (Ferracioli, 2000). Dessa forma, o objetivo deste trabalho é investigar a utilização de um Ambiente

\footnotetext{
*giusepi@bol.com.br

†'laercio@npd.ufes.br
} 
de Modelagem Computacional no estudo de tópicos de Física.

Um Ambiente de Modelagem Computacional é um software que, no contexto deste trabalho, recebeu essa denominação devido ao fato de existir uma proposta pedagógica subjacente à sua utilização onde ele é visto como um Ambiente de Aprendizagem. Assim, este Ambiente pode possibilitar ao aluno a ampliação de sua capacidade em formular perguntas e não simplesmente em encontrar respostas (Ferracioli, 2001).

\section{Referencial Teórico}

A utilização de Ambientes Computacionais na prática da sala de aula pode ser desenvolvida a partir do estudo de tópicos de conteúdos específicos em Ciências com utilização de uma abordagem alternativa valendose do conceito de modelagem.

Esta proposta consiste em levar o aluno a construir um modelo sobre o problema em estudo e, em seguida, representá-lo no computador através de um Ambiente de Modelagem Computacional adequado. Uma vez representado o modelo no Ambiente de Modelagem, este pode ser simulado gerando a possibilidade de ampliação do estudo do problema analisado.

\section{II.1 Sobre Atividades de Modelagem}

De acordo com Bliss \& Ogborn (1989), as atividades de construção de modelos podem ser desenvolvidas de duas maneiras:

- Exploratória, quando o aluno é levado a explorar, no ambiente computacional, um modelo desenvolvido anteriormente por um especialista;

- Expressiva, quando o aluno é solicitado a desenvolver um modelo no Ambiente de Modelagem Computacional.

Neste trabalho, as atividades de construção de modelos foram desenvolvidas em nível exploratório e expressivo. Estas atividades naturalmente envolvem a atividade de raciocínio. Assim, Bliss et al (1992) sugerem que o raciocínio pode ser concebido a partir de três dimensões:

- Quantitativa

- Qualitativa

- Semiquantitativa

O raciocínio quantitativo pode envolver uma variedade de aspectos desde o reconhecimento de relações numéricas simples, seja no trabalho com um conjunto de números através de comparação de tamanhos, até a manipulação de relações algébricas. Essa dimensão de raciocínio envolve o entendimento de quanto a mudança de uma variável afetará outra, por exemplo, no estudo de filas em um supermercado: caso a população dobre quanto esse fator afetará o tempo de espera nas filas do caixa.
O raciocínio qualitativo envolve fazer distinções categóricas e decisões. Isso pode consistir em examinar um conjunto de escolhas ou opções e levar em consideração suas conseqüências: no caso de uma viagem, diferentes meios de transportes podem ser escolhidos. $\mathrm{Ou}$ ainda, considerando-se um dado objetivo, o que é necessário para atingi-lo. Isso pode requerer a observação e consideração de alternativas e a ponderação de evidências: um aluno que deseja passar no vestibular necessita maior dedicação aos estudos.

O raciocínio semiquantitativo envolve a descrição de situações cotidianas onde a direção da mudança de uma parte de um sistema é conhecida mas não o tamanho do efeito desta mudança sobre as demais partes (Ogborn e Miller, 1994). Assim, da observação do comportamento do olho humano, sabe-se que o aumento da intensidade luminosa causa a diminuição da abertura da pupila do olho humano: a análise desse efeito requer o entendimento da direção da relação causal - aumento ou diminuição - mas não o conhecimento dos valores numéricos em relações matemáticas. Neste trabalho, as atividades de modelagem exploratórias e expressivas demandaram do estudante a utilização do raciocínio em nível semiquantitativo

Dessa forma, a construção de modelos em nível semiquantitativo pode ser feita a partir de uma visão sistêmica (Forrester, 1968), em que se procura entender o comportamento de um sistema a partir de relações causais entre as variáveis que o descrevem. Neste sentido, a causalidade desempenha papel fundamental na modelagem semiquantitativa, uma vez que está subjacente no estabelecimento das relações entre as variáveis na argumentação (Bliss et al, 1992).

\section{II.2 Diagramas Causais}

As relações causais entre as variáveis de um sistema em estudo podem ser entendidas e representadas a partir de pares de causa e efeito, denominada Diagrama Causal (Roberts et al, 1983). O exemplo apresentado sobre o comportamento da pupila do olho humano exposta a uma fonte luminosa cuja intensidade pode ser variada, está mostrado na Fig. 1 abaixo através de uma representação por um Diagrama Causal.

\section{Intensidade luminosa $\longrightarrow$ abertura da pupila}

Figura 1. Exemplo de um Par de Causa e Efeito.

Os modelos construídos através de Diagramas Causais podem ser representados no Ambiente de Modelagem Computacional Baseado na Metáfora de Ícones WLinklt. 


\section{II.3 Ambiente de Modelagem Computacional Baseado na Metáfora de Ícones WLinklt}

O WLinkit é um Ambiente de Modelagem Computacional baseado na Metáfora de Ícones para a construção e simulação de modelos dinâmicos em nível semiquantitativo. Nele, é possível construir modelos que re- presentam relações causais entre variáveis relevantes de fenômenos, eventos, objetos do mundo a ser modelado (Sampaio, 1996).

Ao executar-se (rodar) o Ambiente WLinkIt, é apresentado ao usuário a tela do Ambiente de Modelagem conforme Fig. 2.

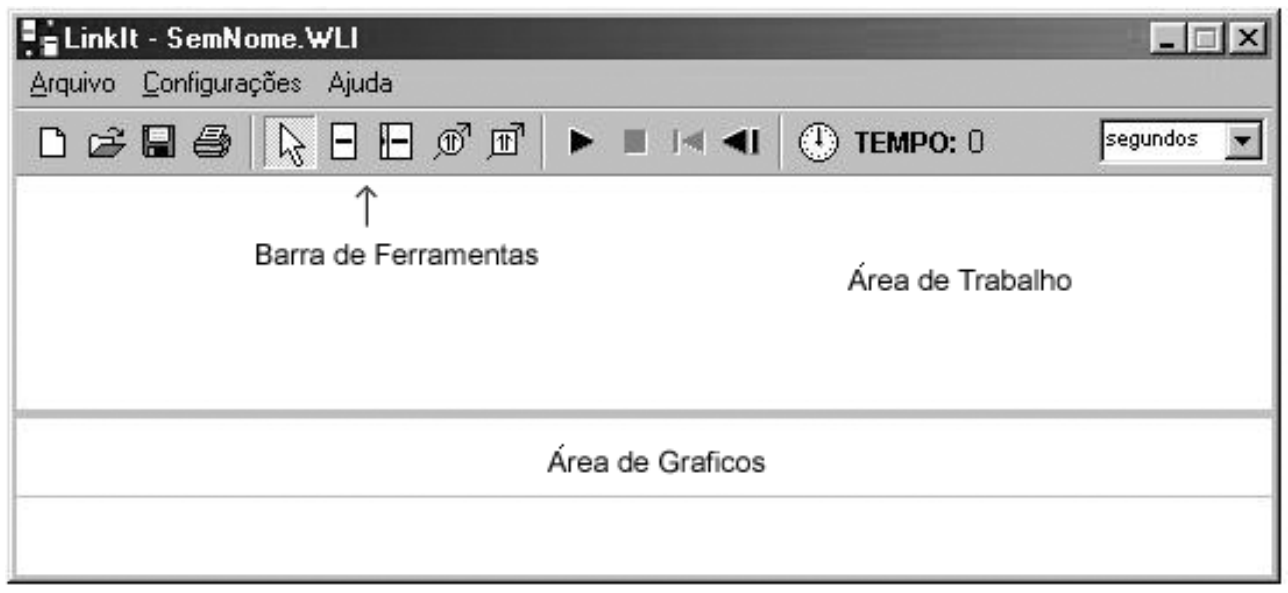

Figura 2. A tela de trabalho do Ambiente de Modelagem Computacional WLinkIt.

A tela é composta de 3 regiões importantes:

Área de trabalho - Esta região nada mais é que um espaço em branco. Ela é a região da tela do computador reservada à construção do modelo pelo usuário;

Área de gráficos - É a região da tela abaixo da Área de Trabalho reservada para a visualização da saída gráfica;

Barra de ferramentas - É a região que contém as ferramentas necessárias para a construção do modelo e está mostrada em detalhes na Fig. 3 abaixo.

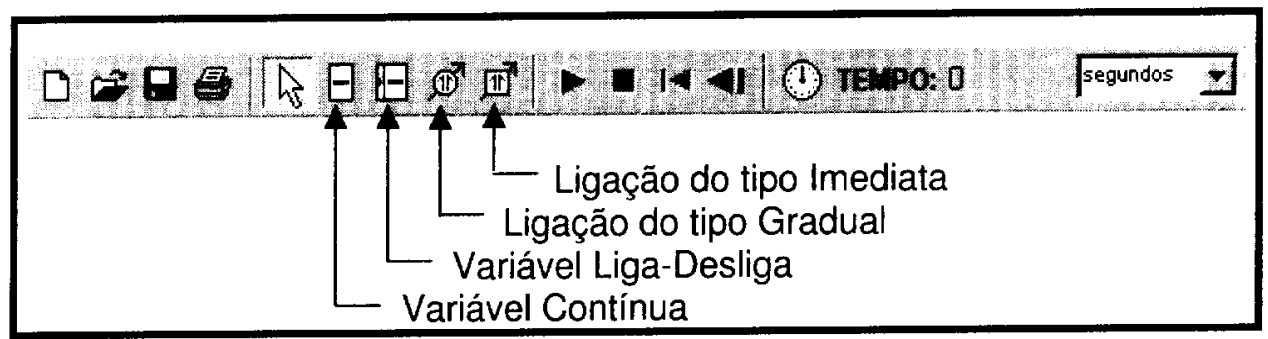

Figura 3. Barra de ferramentas do Ambiente WLinkit.

Em relação à Fig. 3, o primeiro conjunto de ícones apresenta os comandos básicos do Windows. Os ícones seguintes apresentam os elementos de construção do modelo e serão descritos abaixo. Os restantes são usados para a simulação do modelo.

As variáveis, conforme indicadas na Fig. 3, são utilizadas para representar objetos, eventos e variáveis do sistema a ser modelado. No ambiente WLinkit, as variáveis podem ser de dois tipos: Variável Contínua e Variável Liga-Desliga.
- Variável Contínua - Permanece ativa durante todo o tempo de simulação, influenciando suas variáveis dependentes e sendo influenciada pelas variáveis causadoras conectadas a ela.

- Variável Liga-Desliga - Ativa as variáveis dependentes somente quando ultrapassa um determinado valor estipulado pelo usuário (gatilho), sendo influenciada pelas variáveis causadoras conectadas a ela durante todo o tempo de simulação. 
As ligações, conforme indicadas na Fig. 3, permitem definir relações de causa e efeito entre pares de variáveis de um determinado modelo. No ambiente WLinkit os relacionamentos podem ser de dois tipos: Gradual e Imediato.

- Gradual - Representado por um círculo,

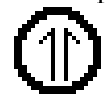

indica que a relação matemática entre um par de variáveis pode ser definida como uma taxa de variação entre as mesmas. Neste tipo de relacionamento, o valor da variável causadora é uma taxa de variação da variável dependente (variável afetada). Uma vez definido o valor da variável causadora, o valor da variável dependente vai crescer ou diminuir gradualmente com o passar do tempo.

- Imediato - Representado por um quadrado

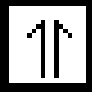

indica uma relação linear entre as variáveis que estão sendo relacionadas. Neste tipo de relacionamento, o valor da variável afetada é imediatamente calculado a partir dos valores das variáveis causadoras.

Uma vez construído o modelo, as propriedades das variáveis e as propriedades das ligações entre elas podem ser definidas através de caixa de diálogo que pode ser acessada efetuando-se um duplo click tanto sobre estas variáveis quanto sobre as ligações.

A construção de modelos neste Ambiente demanda do estudante um raciocínio em nível semiquantitativo. Assim, não é necessário conhecer as relações matemáticas entre as variáveis para a construção de um modelo no Ambiente WLinkIt, pois os cálculos necessários para que ele possa ser simulado são estabelecidos internamente por procedimentos computacionais, livrando o estudante da carga cognitiva exigida pela programação e pelo conhecimento matemático. Em outras palavras, é possível a construção de um modelo icônico que será traduzido em informação simbólica apropriada a partir do qual serão executados cálculos e feitas inferências utilizando-se essas informações cujos resultados serão, por sua vez, traduzidos novamente para a forma icônica.

O modelo construído através do Diagrama Causal mostrado na Fig. 1 pode ser representado no Ambiente WLinkIt conforme pode ser visto na Fig. 4.

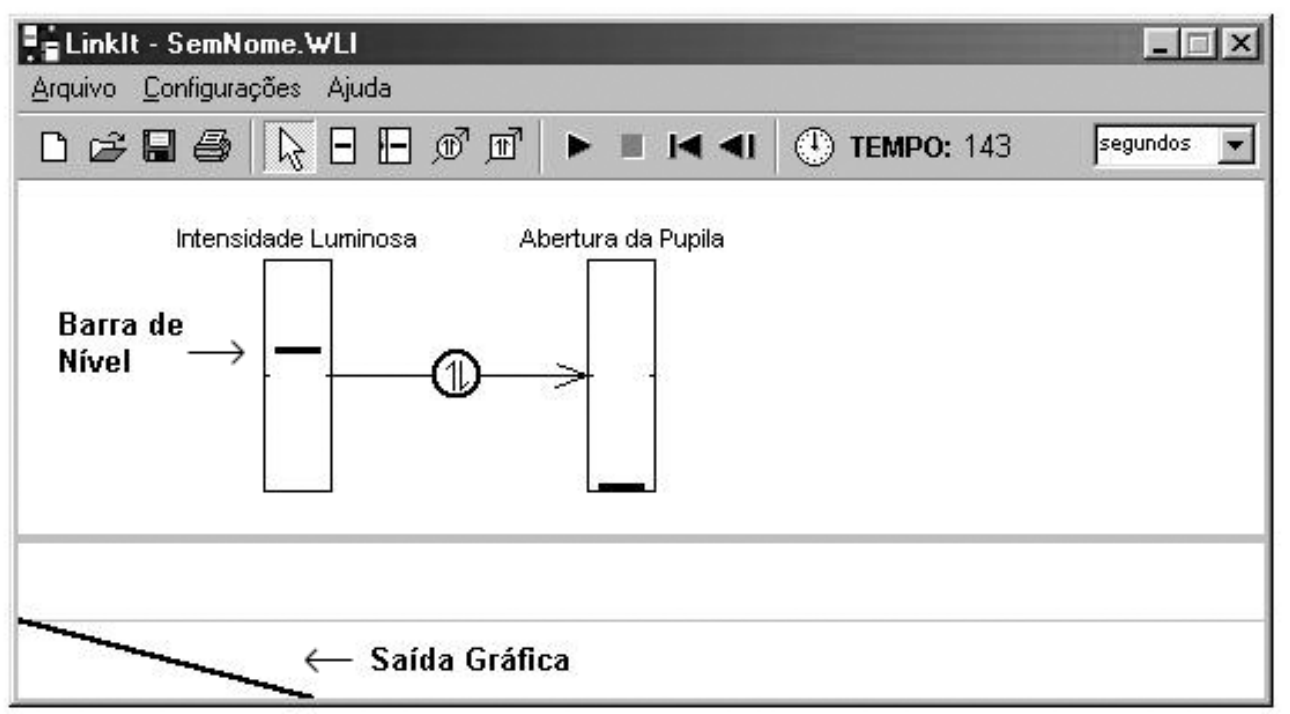

Figura 4. Representação do Diagrama Causal sobre o comportamento da pupila do olho humano e a saída gráfica da variável abertura da pupila.

Enquanto que a versão do modelo no papel revela sua natureza estática, em que é privilegiada uma visão instantânea da realidade física, a sua versão computacional é dinâmica na medida em que o modelo pode ser simulado e os resultados desse processamento possam auxiliar na reestruturação e melhoria do modelo inicial, viabilizando, dessa forma, vislumbrar a evolução temporal dessa mesma realidade física (Ferracioli, 1997). Durante a simulação do modelo, o comportamento de cada variável pode ser visualizado através da barra de nível de cada variável, que é animada, e através da saída gráfica de cada variável que pode ser solicitada pelo usuário e ser construída automaticamente pelo Ambiente WLinkIt.

\section{II.4 Estratégia para a Construção de Modelos}

O processo de elaboração de um modelo e sua representação em um Ambiente de Modelagem Computacional é denominado Processo de Construção do Modelo. Para facilitar o desenvolvimento deste pro- 
cesso, é proposto ao aluno que siga 7 passos básicos apresentados a seguir:

- a definição do sistema a ser estudado;

- escolha do fenômeno de interesse a ser estudado no sistema escolhido;

- listagem das variáveis relevantes para a construção do modelo;

- construção do modelo através de Diagramas Causais;

- representação do modelo no Ambiente de Modelagem Computacional Semiquantitativo WLinkIt;

- simulação do modelo construído;

- validação do modelo a partir da análise de seu comportamento em relação ao comportamento esperado do fenômeno em estudo.

Este procedimento para a construção do modelo, proposto por Camiletti (2001), apresenta uma melhoria em relação ao proposto por Mandinach (1989), que considera somente a listagem de variáveis (laundry list). A justificativa para a inclusão desses passos é promover um maior domínio do problema estudado por parte do aluno.

Este processo pode ser ilustrado para o estudo do comportamento da pupila exposta a uma fonte luminosa cuja intensidade pode ser variada. Os passos estão mostrados abaixo:

- sistema a ser estudado é a pupila do olho;

- fenômeno de interesse é o comportamento da pupila do olho;

- as variáveis relevantes são, a princípio, a intensidade luminosa e a abertura da pupila;

- a construção do modelo através de um Diagrama Causal está mostrada na Fig. 1;

- a representação do modelo no Ambiente WLinkIt e o resultado da simulação, através de saída gráfica, estão mostrados na Fig. 4.

- A validação do modelo é feita pelo usuário, utilizando-se os recursos de animação das variáveis e a saída gráfica do Ambiente WLinkIt. Assim, observando-se a saída gráfica da variável abertura da pupila, pode-se constatar que ela diminuiu quando a intensidade luminosa aumentou, o que está de acordo com a expectativa prévia.

\section{Concepção do Estudo}

Nos últimos anos, tem sido crescente o número de estudos sobre a integração de ambientes computacionais baseados na metáfora de ícones no contexto educacional, no ensino de Física com estudantes de ensino superior (e.g. Santos, Sampaio e Ferracioli, 2001; Camiletti e Ferracioli, 2001; Ferracioli e Sampaio, 2001) e também no ensino de Ciências, de modo geral, com estudantes de ensino fundamental e ensino médio, como relatado por diversos estudos, tais como, Bliss et al.
(1992), Santos e Ogborn (1994), Sampaio (1996), Sampaio e Torres (1999). Esses trabalhos relatam resultados que mostram que a utilização da modelagem e da modelagem computacional baseada na metáfora de ícones no contexto educacional voltado para o estudo de tópicos de Ciências, tem se revelado promissora.

Neste contexto, o presente estudo tem o objetivo de investigar a utilização do Ambiente de Modelagem Computacional Semiquantitativo Baseado na Metáfora de Ícones WLinkIt no estudo de um sistema físico, o sistema mola-massa, a partir do desenvolvimento de atividades exploratórias e expressivas (Camiletti, 2001). Dessa forma, o estudo foi estruturado a partir das seguintes questões básicas de pesquisa:

[1] Como são conceituadas as entidades utilizadas para a construção do modelo e como são as ligações entre elas?

[2] Qual a estratégia utilizada pela dupla para o desenvolvimento da atividade?

[3] Como é a visão do estudante a respeito das variáveis que estão envolvidas no sistema em estudo?

[4] Quais habilidades os estudantes demonstraram durante o desenvolvimento do modelo?

[5] Quais são as dificuldades apresentadas pelos estudantes durante o desenvolvimento do modelo?

\section{III.1 O Contexto}

Para o desenvolvimento desta investigação, foi elaborado um curso de extensão intitulado "Modelagem e Representação de Sistemas Físicos com Ambientes de modelagem Computacional" ministrado em 2 Módulos Instrucionais com duração de 2 horas cada um.

Os dois módulos instrucionais foram organizados como descrito a seguir:

- Módulo 1: Atividades Exploratórias - (02 horas)

Introdução ao estudo do Raciocínio em Nível de Sistema e ao Ambiente de Modelagem Computacional Semiquantitativo WLinkIt

- Módulo 2: Atividades Expressivas - (02 horas)

Modelando e Representando Sistemas com o Ambiente de Modelagem Computacional Semiquantitativo WLinkIt.

O objetivo do Módulo 1 foi introduzir os estudantes no raciocínio em Nível de Sistemas (Forrester, 1968) e ao Ambiente WLinkIt através de atividades de modelagem exploratória.

O Módulo 2 teve o objetivo de levar os estudantes a desenvolver atividades de modelagem expressiva com o Ambiente WLinkIt sobre o Sistema Mola-Massa e sobre Sistema Predador-Presa. Elas se desenvolveram, 
em seqüência, sobre o primeiro e segundo sistemas, respectivamente.

Neste trabalho são relatados somente os resultados das atividades de modelagem expressivas com o Sistema Mola-Massa. Para o desenvolvimento dessa atividade, foi entregue a cada dupla um texto contendo as seguintes informações:

$\mathrm{Na}$ natureza pode-se observar uma série de fenômenos oscilatórios. Considere por exemplo, que uma mola possui uma de suas extremidades presa a um ponto fixo e a outra, presa a um corpo que pode oscilar livremente, conforme mostra a Figura abaixo.

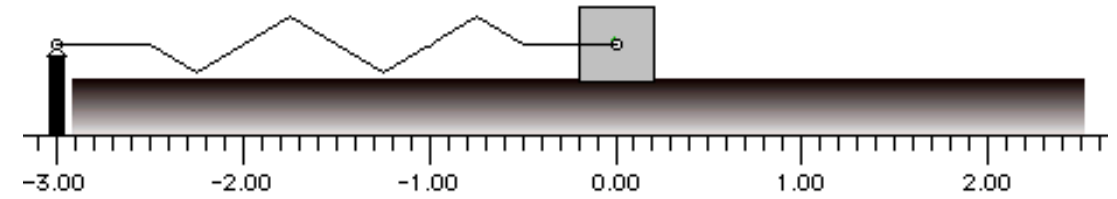

Em seguida, foi solicitado aos estudantes, que construíssem um modelo no computador sobre esse sistema, discutindo as idéias com o colega de dupla e explicando o que estava sendo feito, em voz alta.

\section{III.2 A Modelagem do Sistema Mola-Massa no Ambiente WLinkIt}

Será apresentado agora o processo de construção do modelo que deveria ser desenvolvido pelo estudante du- rante a atividade de modelagem expressiva.

O sistema a ser estudado é o bloco preso a uma mola;

O fenômeno de interesse é o movimento do bloco;

As variáveis relevantes para o estudo do movimento do bloco são a posição, a velocidade e a aceleração;

A construção do modelo diretamente no Ambiente WLinkIt e o resultado da simulação, através de saída gráfica, estão mostrados na Fig. 5.

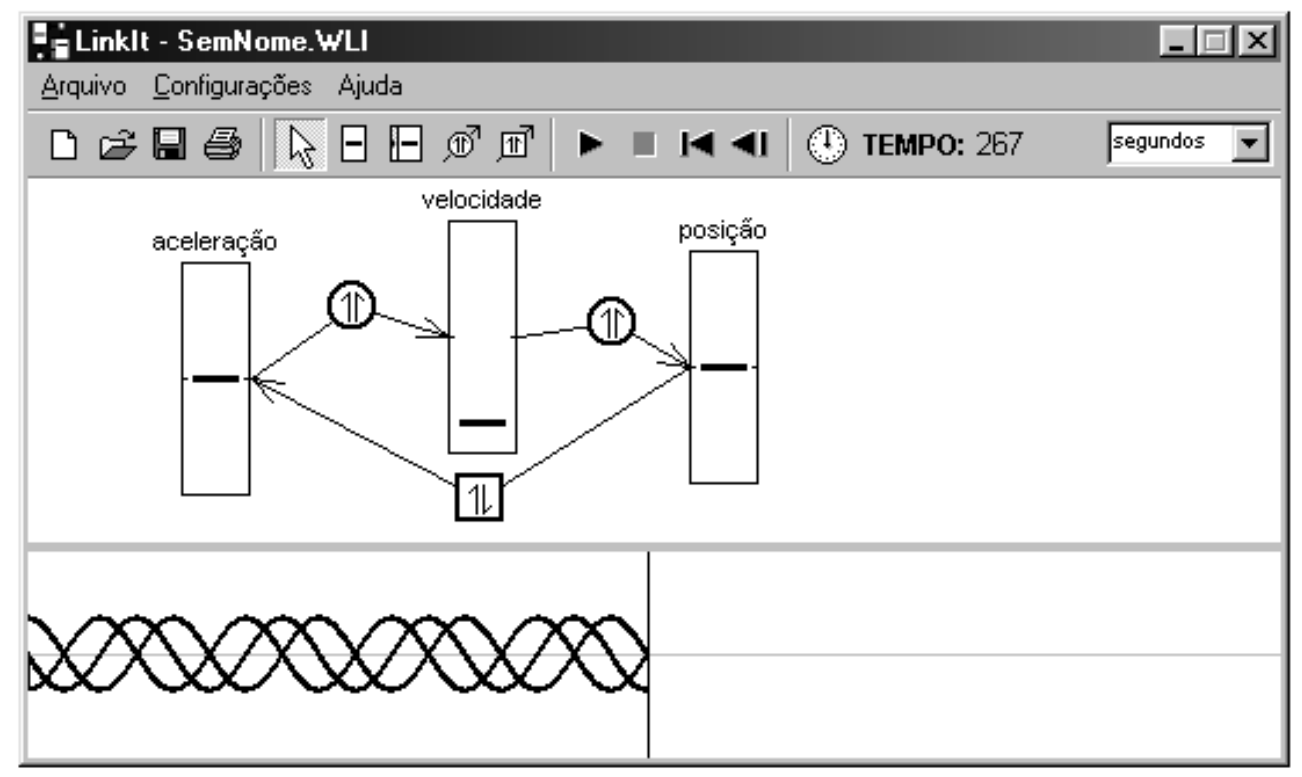

Figura 5. O modelo do sistema mola-massa no Ambiente WLinkIt.

Neste modelo, a aceleração é vista como a causa da velocidade, e esta como a causa da posição. A aceleração depende da posição de modo que, quanto maior, positivamente, for a posição, maior, negativamente, é a aceleração. Assim, a aceleração, assumindo valores negativos, faz a velocidade diminuir e esta por sua vez faz a posição diminuir, a qual, por sua vez, faz a aceleração aumentar gerando, assim, um movimento oscilatório.

\section{III.3 Amostragem}

Participaram do curso estudantes universitários do quarto período da área de Ciências Exatas e Enge- 
nharia da Universidade Federal do Espírito Santo. Os alunos foram agrupados em duplas e as aulas foram ministradas exclusivamente para cada dupla. No total, 6 duplas desenvolveram as atividades dos Módulos 1 e 2 .

\section{III.4 Coleta de Dados}

Todas as atividades desenvolvidas pelos estudantes foram vídeo filmadas e o material escrito foi recolhido.

\section{III.5 Dados}

Os dados considerados para a análise consistiram no material escrito pelos estudantes; a seqüência de versões do modelo construído pelas duplas e das argumentações dos estudantes foi gravada em vídeo.

\section{Análise de Dados}

Os dados são de natureza qualitativa e o instrumento utilizado para a análise dos dados foi a Rede Sistêmica (Bliss et al., 1983), devido à possibilidade de estruturação de categorias de uma forma mais abrangente e complexa. De acordo com Ogborn (1994), uma rede sistêmica pode ser vista como uma gramática, independentemente do contexto que define uma 'linguagem' construída para descrever os dados. Os elementos básicos de uma Rede Sistêmica são:

- Colchete - usado para representar conjunto de escolhas exclusivas;

- Chave - usada para representar conjunto de escolhas que ocorrem simultaneamente.

O aspecto analisado foi o Processo de Construção do Modelo, descrito na seção II.4, e a rede sistêmica construída para a análise deste processo está mostrada na Fig. 6. Ela é constituída de dois aspectos básicos: Descrição e Análise do processo de desenvolvimento da atividade expressiva. Estes aspectos são constituídos de categorias que refletem as características de todas as duplas e estão explicitadas através de duas redes sistêmicas que podem ser vistas na parte esquerda das Figs. 7 e 8, respectivamente. Portanto, a rede sistêmica final reflete o conjunto dos aspectos de Descrição e Análise do Processo de Construção do Modelo de todas as duplas.

As Figs. 7 e 8 apresentam também, na sua parte direita, um quadro-resumo das atividades desenvolvidas pelas duplas, que ser' a descrito mais adiante.

A leitura da rede sistêmica é feita de acordo com a definição do colchete e da chave. Considerandose, como exemplo, o aspecto Modelo Final, na parte da rede sistêmica relativa ao aspecto de Descrição do Processo de Construção do Modelo, a chave significa que dois aspectos foram levados em consideração simultaneamente: comportamento das variáveis do modelo $\mathrm{e}$ número de variáveis utilizadas no modelo. O colchete à frente do aspecto comportamento fornece a informação de que as variáveis do modelo final da dupla em questão apresentaram comportamento oscilatório ou não oscilatório. O colchete à frente do aspecto número de variáveis fornece a informação de que o modelo final da dupla era constituído de duas variáveis ou mais de três variáveis.

Um outro ponto importante a ser observado em uma rede sistêmica é o da posição relativa dos termos envolvidos. Os termos mais à esquerda da rede, por exemplo o Modelo Final, fornecem informações mais gerais sobre os dados, enquanto que os termos mais à direita da rede, por exemplo, número de variáveis, apresentam um nível de detalhamento maior onde as informações são mais próximas dos dados brutos.

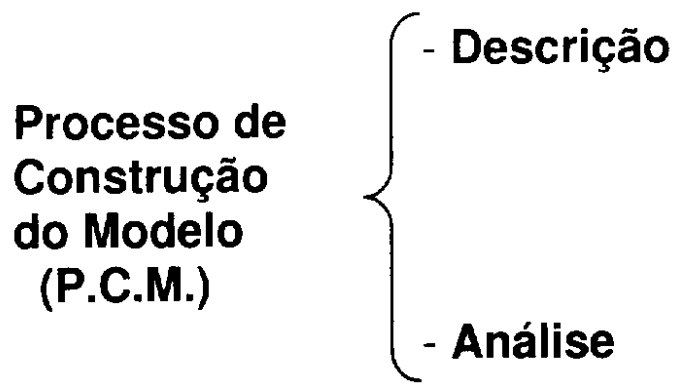

Figura 6. Rede Sistêmica para a Análise dos Dados.

Em relação aos quadros-resumo anexos às redes sistêmicas nas Figs. 7 e 8, eles proporcionam uma leitura dos aspectos de Descrição e Análise do Processo de Construção do Modelo do sistema mola-massa. No quadro, uma posição marcada significa que a dupla em questão apresentou aquele tipo de comportamento.

\section{IV.1 Exemplar de Análise de uma Dupla}

A análise do Processo de Construção do Modelo pode ser feita a partir da leitura dos quadros anexos às redes sistêmicas das Figs. 7 e 8 . Uma leitura por colunas proporciona uma visualização do comportamento de cada dupla, enquanto que uma leitura por linhas proporciona a visualização do comportamento de todas as duplas em um aspecto específico.

Assim, a título de ilustração, é apresentada a análise do Processo de Construção do Modelo da Dupla 01. A Fig. 9 apresenta o modelo final construído por esta dupla.

Em relação aos aspectos de Descrição, através da Fig. 7, pode-se observar que no aspecto:

- Início da Atividade, a dupla realizou a leitura do texto e não seguiu os passos sugeridos no Módulo 1; 
- Postura de Trabalho, o modelo foi desenvolvido diretamente no computador;

- Elementos de Modelagem, as variáveis foram concebidas como quantidades e as ligações entre elas foram concebidas como causais;

- Versão do Modelo, após a construção de uma versão, a dupla realizou a simulação e concluiu que o comportamento das variáveis não estava de acordo com o esperado. Assim, o modelo sofreu alterações se transformando em uma nova versão. Este processo foi ocorrendo até a dupla chegar á versão final;

\section{Sistema Mola-Massa}

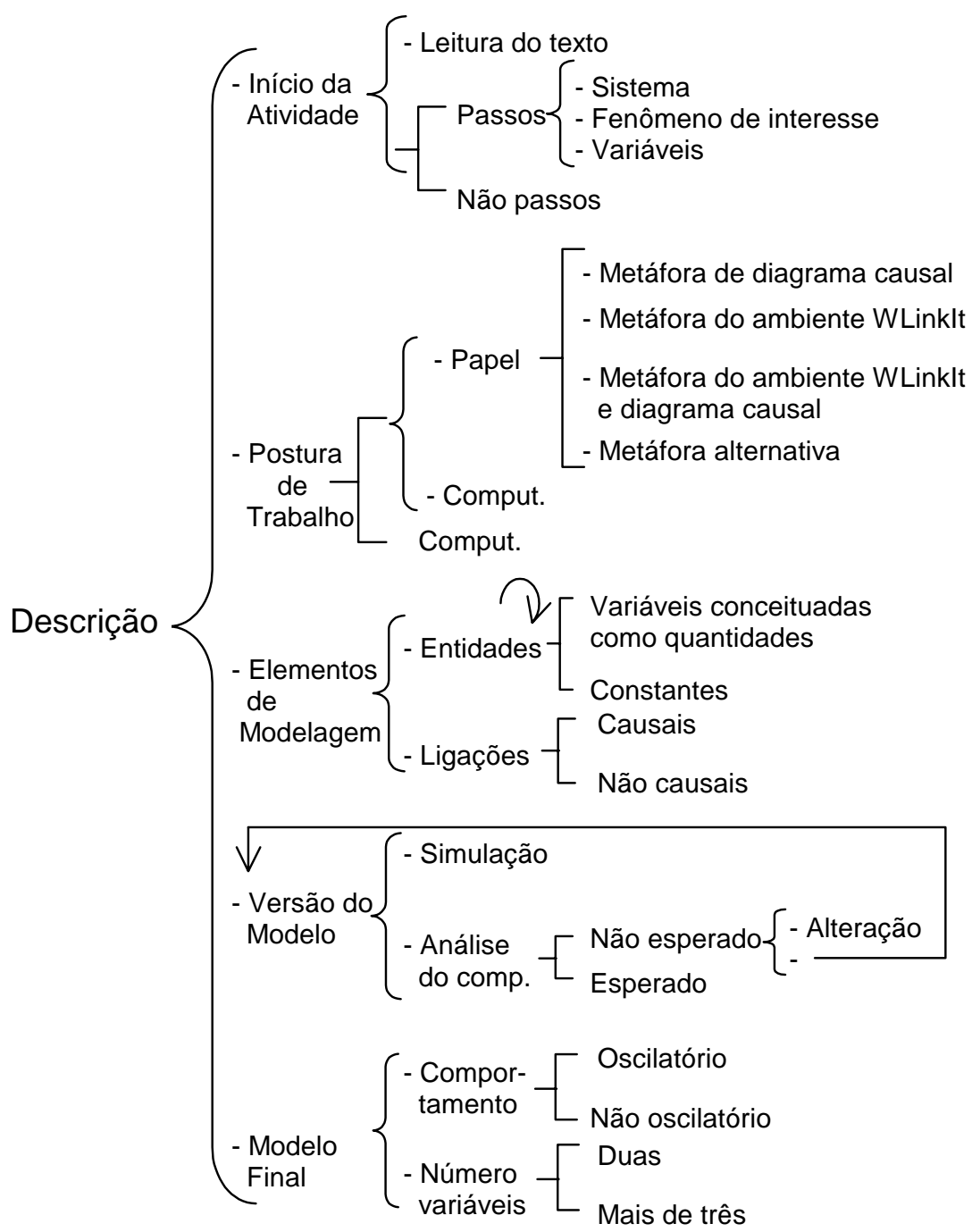

\begin{tabular}{|c|c|c|c|c|c|c|}
\hline \multicolumn{7}{|c|}{ D u p I a s } \\
\hline 01 & 02 & 03 & 04 & 05 & 06 & Total \\
\hline $\mathrm{x}$ & $\mathrm{x}$ & $\mathrm{x}$ & $\mathrm{x}$ & $\mathrm{x}$ & $\mathrm{x}$ & 06 \\
\hline & $\mathrm{x}$ & $\mathrm{x}$ & $\mathrm{x}$ & & $\mathrm{x}$ & 04 \\
\hline & $\mathrm{x}$ & $\mathrm{x}$ & $\mathrm{x}$ & & $\mathrm{x}$ & 04 \\
\hline & $\mathrm{x}$ & $\mathrm{x}$ & $\mathrm{x}$ & $\mathrm{x}$ & $\mathrm{x}$ & 05 \\
\hline $\mathrm{x}$ & & & & & & 01 \\
\hline & & $\mathrm{x}$ & & & & 01 \\
\hline & & & & & $\mathrm{x}$ & 01 \\
\hline & & & & $\mathrm{x}$ & & 01 \\
\hline & & & & & $\mathrm{x}$ & 01 \\
\hline & & $\mathrm{x}$ & & $\mathrm{x}$ & $\mathrm{x}$ & 03 \\
\hline $\mathrm{x}$ & $\mathrm{x}$ & & $\mathrm{x}$ & & & 03 \\
\hline
\end{tabular}

\begin{tabular}{|c|c|c|c|c|c|c|}
\hline$x$ & $x$ & $x$ & $x$ & $x$ & $x$ & 06 \\
\hline & $x$ & & $x$ & $x$ & & 03 \\
\hline$x$ & & $x$ & $x$ & & & 03 \\
\hline & $x$ & & & $x$ & $x$ & 03 \\
\hline
\end{tabular}

\begin{tabular}{|c|c|c|c|c|c|c|}
\hline$x$ & $x$ & $x$ & $x$ & $x$ & $x$ & 06 \\
\hline$x$ & $x$ & $x$ & $x$ & $x$ & $x$ & 06 \\
\hline & & & & & & - \\
\hline
\end{tabular}

\begin{tabular}{|c|c|c|c|c|c|c|}
\hline$x$ & $x$ & $x$ & $x$ & & & 04 \\
\hline & & & & $x$ & $x$ & 02 \\
\hline$x$ & $x$ & $x$ & $x$ & & & 04 \\
\hline & & & & $x$ & $x$ & 02 \\
\hline
\end{tabular}

Figura 7. Rede Sistêmica e Quadro-Resumo dos aspectos de Descrição da Atividade de Modelagem Expressiva com o Sistema Mola-Massa. 
Sistema Mola-Massa

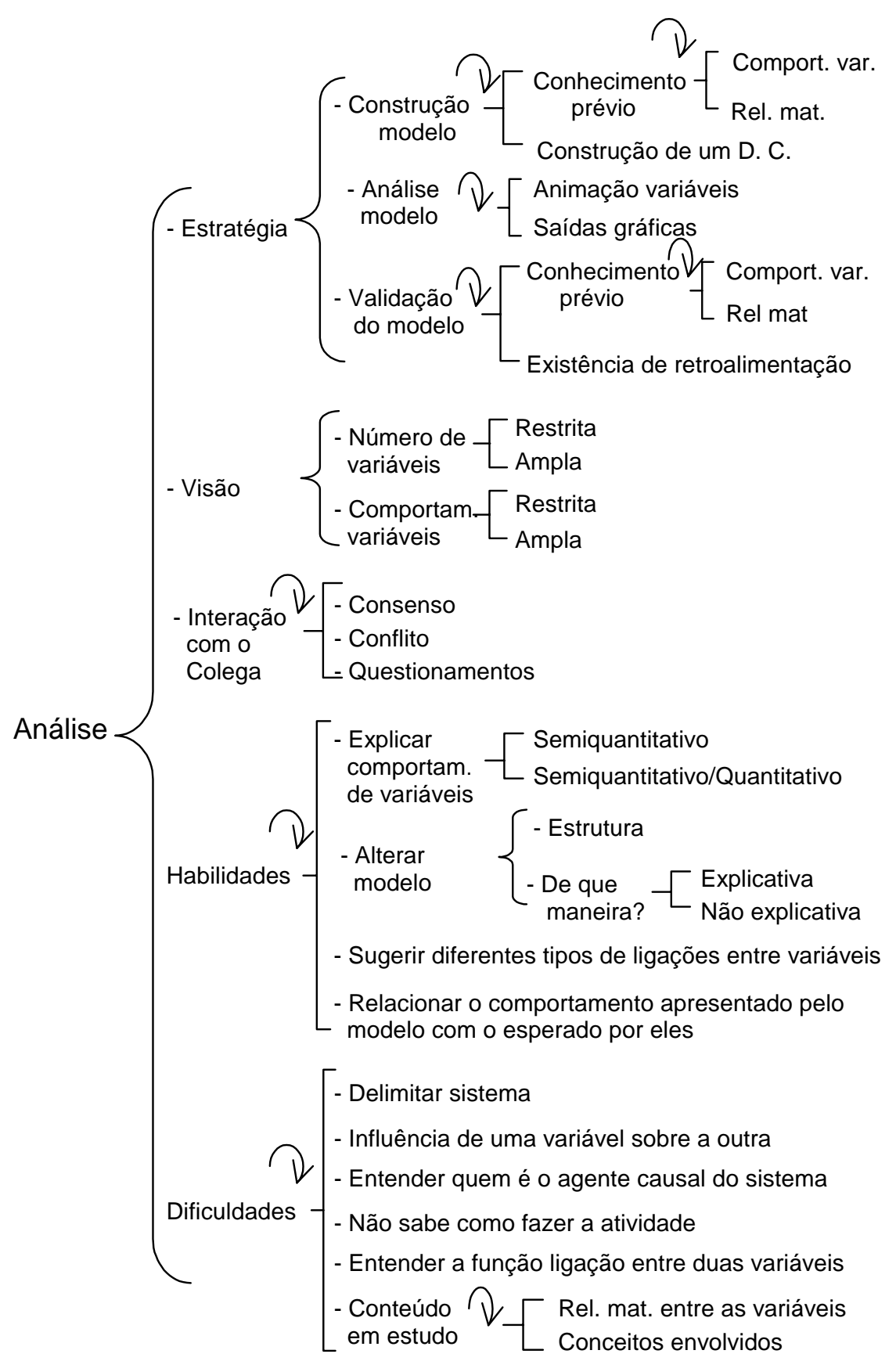

\begin{tabular}{|c|c|c|c|c|c|c|}
\hline \multicolumn{7}{|c|}{$D$ u p I a s } \\
\hline 01 & 02 & 03 & 04 & 05 & 06 & Total \\
\hline $\mathrm{x}$ & $\mathrm{x}$ & $\mathrm{x}$ & $\mathrm{x}$ & $\mathrm{x}$ & $\mathrm{x}$ & 06 \\
\hline & & & $\mathrm{x}$ & & $\mathrm{x}$ & 02 \\
\hline & & $\mathrm{x}$ & & & & 01 \\
\hline $\mathrm{x}$ & $\mathrm{x}$ & $\mathrm{x}$ & $\mathrm{x}$ & $\mathrm{x}$ & $\mathrm{x}$ & 06 \\
\hline $\mathrm{x}$ & & $\mathrm{x}$ & $\mathrm{x}$ & $\mathrm{x}$ & $\mathrm{x}$ & 05 \\
\hline $\mathrm{x}$ & $\mathrm{x}$ & $\mathrm{x}$ & $\mathrm{x}$ & $\mathrm{x}$ & $\mathrm{x}$ & 06 \\
\hline $\mathrm{x}$ & & & & & & 01 \\
\hline & $\mathrm{x}$ & & & & & 01 \\
\hline
\end{tabular}

\begin{tabular}{|c|c|c|c|c|c|c|}
\hline$x$ & $x$ & & & & & 02 \\
\hline & & $x$ & $x$ & $x$ & $x$ & 04 \\
\hline & & & & $x$ & & 01 \\
\hline$x$ & $x$ & $x$ & $x$ & & $x$ & 05 \\
\hline
\end{tabular}

\begin{tabular}{|c|c|c|c|c|c|c|}
\hline$x$ & $x$ & $x$ & $x$ & $x$ & $x$ & 06 \\
\hline & & $x$ & $x$ & $x$ & $x$ & 04 \\
\hline$x$ & $x$ & $x$ & $x$ & $x$ & $x$ & 06 \\
\hline
\end{tabular}

\begin{tabular}{|c|c|c|c|c|c|c|}
\hline & $x$ & & $x$ & $x$ & $x$ & 04 \\
\hline$x$ & & $x$ & & & & 02 \\
\hline$x$ & $x$ & $x$ & $x$ & $x$ & $x$ & 06 \\
\hline$x$ & $x$ & $x$ & $x$ & & & 04 \\
\hline & & & & $x$ & $x$ & 02 \\
\hline$x$ & & & $x$ & & & 02 \\
\hline$x$ & $x$ & $x$ & $x$ & $x$ & $x$ & 06 \\
\hline
\end{tabular}

\begin{tabular}{|c|c|c|c|c|c|c|}
\hline & $x$ & $x$ & & & $x$ & 03 \\
\hline & & $x$ & $x$ & & $x$ & 03 \\
\hline & $x$ & & & & & 01 \\
\hline & & & & $x$ & $x$ & 02 \\
\hline & & & & $x$ & & 01 \\
\hline$x$ & & & & & & 01 \\
\hline & & & & & $x$ & 01 \\
\hline
\end{tabular}

Figura 8. Rede Sistêmica e Quadro-Resumo dos aspectos de Análise da Atividade de Modelagem Expressiva com o Sistema Mola-Massa.

- Modelo Final apresentou comportamento oscilatório com utilização de somente duas variáveis, este último fato revelando uma visão definida como restrita das variáveis envolvidas no modelo.

Em relação aos aspectos de Análise, através da Fig.
8, pode-se observar que no aspecto:

- Estratégia, a dupla baseou-se no conhecimento prévio sobre o comportamento das variáveis. A análise do modelo foi feita através da animação das variáveis 


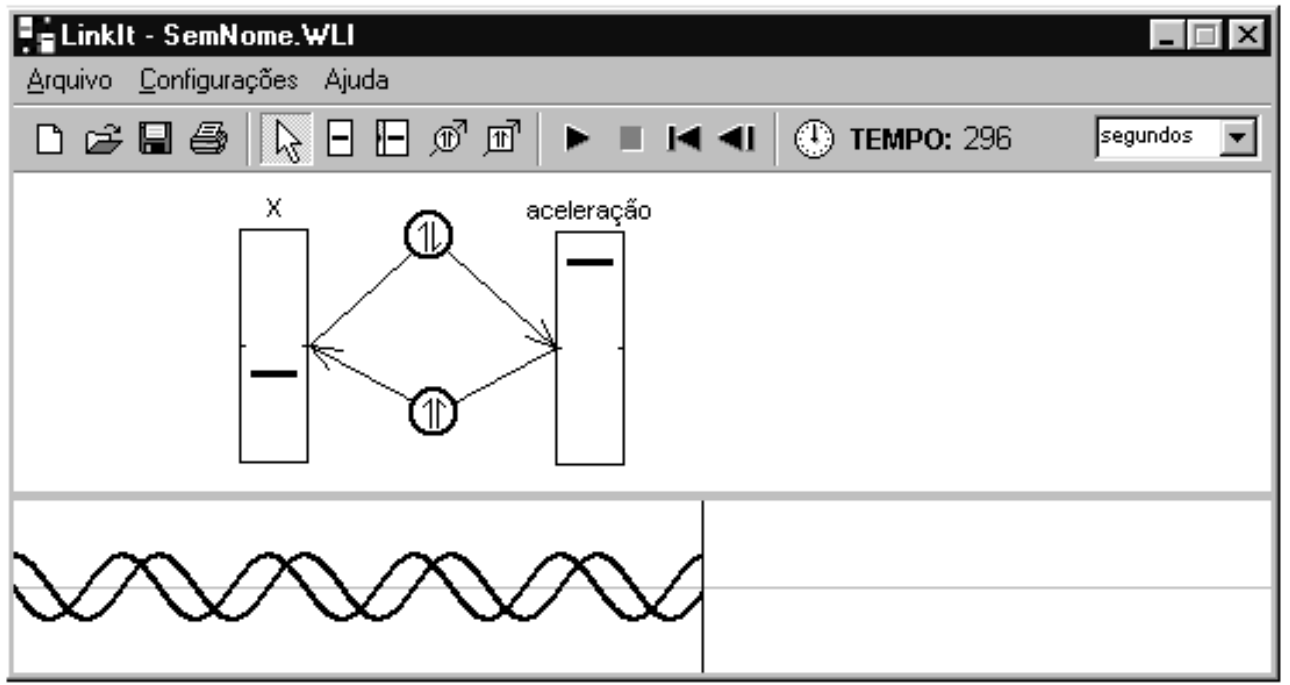

Figura 9. Modelo Final da Dupla 01. Na Área de Gráficos do Ambiente WLinkIt, está mostrada a saída gráfica das duas variáveis do modelo. Para a simulação deste modelo, foi dado um valor inicial positivo para a variável $\mathrm{X}$ e valor zero para a variável aceleração.

e da saída gráfica. Já a validação do modelo foi baseada no conhecimento prévio sobre comportamento das variáveis e no conhecimento sobre as relações matemáticas entre as variáveis;

- Visão, a dupla apresentou uma visão restrita em relação ao número de variáveis consideradas para a construção do modelo e uma visão ampla em relação ao comportamento das variáveis;

- Interação com o colega, os estudantes da dupla apresentaram consenso, quando havia a concordância de ambos os estudantes em relação ao processo de construção do modelo, e questionamentos entre si, quando diferentes visões sobre o processo de construção do modelo eram debatidas pelos estudantes da dupla. Esta dupla não apresentou conflito, situação caracterizada neste estudo quando cada estudante tinha sua própria opinião, diferente da do colega, podendo ou não se chegar a um consenso ao final da interação;

- Habilidades, a dupla explicou o comportamento das variáveis em nível semiquantitativo e em alguns momentos em nível quantitativo, alterou o modelo, sugeriu que deveria haver diferentes tipos de ligações entre as variáveis e relacionou o comportamento apresentado pelo modelo com o esperado por eles;

- Dificuldades, a dupla encontrou dificuldades para utilizar relações matemáticas entre as variáveis que foram consideradas por eles.

A partir dos quadros-resumo, é possível obter também uma leitura sobre o comportamento das duplas em cada aspecto considerado na rede. Como exemplo, considerando-se as Habilidades apresentadas pelas duplas, todos os estudantes foram capazes de explicar o comportamento das variáveis em nível semiquantitativo e, em alguns casos, foram também capazes de utilizar argumentos quantitativos para dar suporte à explicação elaborada por eles.

\section{Discussão e Conclusão}

A conclusão sobre o Processo de Construção do Modelo será feita respondendo as questões básicas de pesquisa deste trabalho, descritas na seção III. A resposta de cada questão é baseada nos quadros-resumo mostrados nas Figs. 7 e 8.

V.1 Como são conceituadas as entidades utilizadas para a construção do modelo e como são concebidas as ligações entre elas?

As entidades selecionadas para a construção dos modelos foram variáveis e constantes. As constantes foram listadas para o desenvolvimento da atividade de modelagem expressiva mas não foram utilizadas ou incluídas no modelo construído. Assim, os modelos foram constituídos de variáveis.

Quanto à conceituação das variáveis, todas as duplas as conceberam como uma quantidade, ou seja, o nome da variável traduzia um atributo quantificável da mesma, sendo exemplo deste tipo de conceituação 'velocidade'. Para os Físicos conceber um sistema através de variáveis e de suas relações pode ser um procedimento natural, no entanto não é um procedimento simples para estudantes entre 12 e 14 anos de idade. Ogborn (1992) comenta que existe evidência de que estudantes desta faixa etária, quando solicitados a construir modelos em um ambiente de modelagem computacional semiquantitativo, têm a tendência de ver o mundo como construído de objetos e eventos ao invés de variáveis quantificáveis. Ainda em relação a este aspecto, o autor argumenta que, no caso de se trabalhar com modelagem computacional com alunos entre 8 e 12 anos de idade, parece que a utilização de um ambiente de modelagem 
computacional que permita a construção de modelos que utilizam variáveis conceituadas como objetos e/ou eventos é mais adequada. Esses tipos de ambientes são baseados, por exemplo, nas regras dos autômatos celulares de Von Neumann (Boohan, 1994). A razão disso é que, segundo o autor, as relações entre as variáveis conceituadas como objetos são, na maioria dos casos, simples e intuitivas.

Em relação ao estabelecimento das ligações entre as variáveis, é considerado que o aluno estabeleceu uma relação causal entre duas variáveis quando concebeu que uma variável mudou devido à ação da outra. Assim, no desenvolvimento do modelo sobre o sistema molamassa, metade das duplas estabeleceu relações causais entre as variáveis.

\section{V.2 Qual a estratégia utilizada pela dupla para o desenvolvimento da atividade de modelagem expressiva?}

Esta questão é respondida a partir de 3 outras, que refletem os aspectos relacionados à análise do processo de desenvolvimento da atividade expressiva da rede sistêmica apresentada na Fig. 8: estratégia de construção do modelo, estratégia de análise do modelo e estratégia de validação do modelo.

\section{Qual a estratégia de construção do modelo uti- lizada pela dupla?}

Em nível de estrutura, o processo de construção de um modelo no Ambiente de Modelagem Computacional WLinkIt consiste na escolha das variáveis relevantes e no estabelecimento de relações entre elas. Assim, a estratégia usada para a construção dos modelos pelas duplas foi predominantemente baseada no conhecimento que os estudantes já possuíam sobre os sistemas em estudo, sendo que duas duplas também utilizaram o conhecimento das relações matemáticas entre as variáveis.

Qual a estratégia utilizada para a análise do comportamento do modelo?

Os recursos do Ambiente de Modelagem Computacional Semiquantitativo Icônico WLinkIt disponíveis para a análise do comportamento do modelo são a animação das variáveis e da saída gráfica. Todas as duplas utilizaram a animação das variáveis para a análise nas duas atividades. A saída gráfica como recurso de análise do comportamento das variáveis do modelo foi utilizada por quase todas as duplas. É importante ressaltar que durante as atividades desenvolvidas no Módulo 1, foi incentivado o uso da saída gráfica para a análise do comportamento das variáveis do modelo.

Um aspecto a ser considerado é que das quatro duplas que construíram um modelo que apresentou comportamento oscilatório e analisaram esse comportamento através das saídas gráficas, três delas foram capazes de identificar que o comportamento oscilatório apresentado pelo modelo não estava de acordo com o esperado por eles: duas duplas argumentaram que a diferença de fase entre as variáveis não estava de acordo com o que esperavam, e outra observou que a amplitude das variáveis aumentava continuamente. Embora os alunos não tenham conseguido concluir qual o problema com o modelo construído, através da utilização da saída gráfica eles puderam constatar a existência desse problema. A outra dupla, cujo modelo também apresentou comportamento oscilatório e não utilizou a saída gráfica, sequer considerou a possibilidade de o comportamento do modelo não estar adequado. Dessa forma, este fato corrobora os resultados relatados por Camiletti \& Ferracioli (2001) de que a saída gráfica se constituiu em um recurso mais poderoso para a validação do comportamento do modelo do que simplesmente a utilização da animação das variáveis.

Um outro aspecto sobre a utilização da saída gráfica é em relação ao nível de argumentação atingido pelo aluno. Os alunos das duplas 01 e 04 em algum momento da construção do modelo teceram comentários baseados em possíveis valores que as variáveis poderiam ter, uma em relação às outras, ou seja, foram capazes de apresentar um argumento em nível quantitativo sobre o comportamento das variáveis do modelo construído em um Ambiente de Modelagem Computacional Semiquantitativo, o que de certa forma os auxiliou na conclusão de que o modelo não apresentava o comportamento que eles esperavam. Assim sendo, a saída gráfica parece prover o estudante de um senso de quantificação das variáveis quando observa o comportamento delas a partir da comparação dos valores de uma em relação às outras.

Este resultado parece dar sustentação ao relato de Santos, Sampaio e Ferracioli (2001), que trabalharam com a hipótese de que o raciocínio em nível semiquantitativo seria precursor do raciocínio em nível quantitativo. De qualquer modo, como comentam os autores, esta hipótese de trabalho demanda o desenvolvimento de estudos sistemáticos sobre a ponte entre estes dois tipos de raciocínio quanto à modelagem computacional.

Qual a estratégia utilizada para a validação do modelo?

Para a validação do modelo, todas as duplas utilizaram o conhecimento prévio sobre o comportamento das variáveis na atividade expressiva. A dupla 01 também fez a respeito das relações matemáticas entre as variáveis, e a dupla 02 atribuiu o comportamento apresentado pelas duas variáveis à existência de uma retroalimentação entre elas.

Em relação à validação do modelo, somente uma dupla considerou que o modelo construído estivesse de acordo com o que eles esperavam, enquanto que as demais concluíram que não. 


\section{V.3 Como é a visão do estudante a respeito das variáveis que estão envolvidas no sistema em es- tudo?}

Em relação ao número de variáveis envolvidas, a maioria das duplas demonstrou uma visão ampla, definida neste estudo como a seleção de mais de duas variáveis para o desenvolvimento do modelo sobre o sistema. Isso pode ser um indício de que alunos de nível universitário provenientes da área de ciências exatas, quando solicitados a construir um modelo sobre tópicos de Física, são capazes de identificar um número de entidades suficientes para a construção de um modelo sobre o tópico em estudo.

Em relação ao comportamento das variáveis do modelo a maioria das duplas apresentou uma visão ampla, definida neste estudo como a situação em que os alunos conceberam as variáveis envolvidas no modelo como disponíveis a assumir valores positivos e negativos. Este resultado parece indicar que os alunos do nível universitário provenientes da área de ciências exatas são capazes de conceber uma entidade representada no Ambiente WLinkIt como algo a que se pode atribuir um valor numérico, e que este valor pode variar entre positivo e negativo.

\section{V.4 Quais habilidades os estudantes demon- straram durante o desenvolvimento do modelo?}

As habilidades apresentadas variaram de dupla para dupla. Todas as duplas foram capazes de construir uma versão do modelo, realizar sua simulação e emitir uma opinião, dizendo se o comportamento apresentado pelo modelo estava ou não de acordo com o que esperavam.

Quatro foram capazes de fornecer explicações em nível semiquantitativo sobre o comportamento das variáveis, ou seja, foram capazes de explicar o que ocorre com uma variável quando a outra varia. Este resultado parece indicar que os alunos foram capazes de raciocinar em nível semiquantitativo para o desenvolvimento da atividade de modelagem expressiva com o sistema mola-massa.

Duas duplas foram capazes também, em algum momento da construção do modelo, de tecer comentários baseados em possíveis valores que as variáveis deveriam ter, uma em relação à outra, ou seja, foram capazes de apresentar um argumento em nível quantitativo sobre o comportamento das variáveis do modelo construído em um Ambiente de Modelagem Computacional Semiquantitativo. Este resultado parece dar sustentação ao relato de Santos, Sampaio e Ferracioli (2001) que trabalharam com a hipótese de que o raciocínio em nível semiquantitativo seria precursor do raciocínio em nível quantitativo.

Todas as duplas foram capazes de alterar o modelo, inserindo ou retirando variáveis e ligações. A maioria foi capaz de explicar essas alterações feitas no modelo, o que indica que elas apresentaram um certo grau de entendimento sobre que estavam fazendo, não adotando uma estratégia de simples tentativa e erro para a construção do modelo.

V.5 Quais são as dificuldades apresentadas pelos estudantes durante o desenvolvimento do modelo?

Para o desenvolvimento da atividade de modelagem expressiva com o sistema mola-massa, as duplas apresentaram uma série de dificuldades, algumas relacionadas à utilização do Ambiente de Modelagem Computacional, outras relacionadas à utilização do raciocínio em nível de sistemas e, em menor escala, ao conteúdo em estudo.

Em relação à utilização do Ambiente de Modelagem Computacional Semiquantitativo Icônico WLinkIt, foi considerado que a dupla teve dificuldade de entender a função da ligação entre duas variáveis, quando ao estabelecer uma ligação entre elas pareciam não entender qual seria o comportamento que seria gerado pela estrutura construída. Assim, parece que a dupla 05, que apresentou este tipo de dificuldade, não possuía um bom nível de entendimento sobre a função da ligação entre duas variáveis do Ambiente de Modelagem Computacional WLinkIt.

Em relação à utilização do raciocínio em nível de sistemas para a construção do modelo, três duplas encontraram dificuldades para entender como uma variável influencia a outra, ou seja, encontraram dificuldades em conceber uma variável como sendo responsável pela variação da outra variável. Uma dupla apresentou dificuldades em relação ao entendimento do agente causal do sistema, comentando em alguns momentos que o agente responsável por fazer o bloco oscilar seria a mola e, em outros momentos, que seria a força. Esta dificuldade pode influenciar na construção do modelo sobre o sistema em estudo, uma vez que põe em dúvida quais são as variáveis que devem ser responsáveis pela variação de outra variável.

Três duplas encontraram dificuldades para a delimitação do sistema a ser estudado. Esta habilidade é fundamental para que o aluno tenha clareza sobre quais aspectos de relevância considerar na construção do modelo, caso contrário alguns aspectos considerados e traduzidos na forma de variáveis podem não ter importância para o modelo, e ainda o aluno pode imaginar um comportamento não adequado para determinada variável.

Portanto, existe um indício de que a dificuldade em delimitar o sistema a ser estudado é uma herança do ensino tradicional, uma vez que, de modo geral, não é observado, de maneira apropriada, o processo de delimitação do sistema no momento do estudo de um determinado tópico: assume-se que o arranjo e manipulação do sistema, com o estabelecimento das condições iniciais não são parte do estudo, uma vez que são fornecidos a priori sem uma discussão adequada. Este 
fato é continuamente reafirmado através da proposição de problemas apresentados ao final de cada capítulo dos livros de Física: massas são comprimidas e colocadas em movimento sem que sejam mencionadas as fontes para o estabelecimento desses conceitos. (Ferracioli, 1994).

Em relação a não saber como realizar a atividade, duas duplas não tinham muito claro o que de fato estava tentando fazer durante o desenvolvimento da atividade de modelagem expressiva com o sistema mola-massa. Simplesmente as variáveis foram sendo incorporadas ao modelo sem o compromisso com um objetivo final.

Em relação à dificuldade com os conceitos envolvidos, uma dupla considerou a variável força como sendo importante para a construção do modelo, mas não sabia se ela representava a força da mola sobre o bloco, a força peso ou a força de atrito. Esta dupla encontrou uma grande dificuldade para desenvolver o modelo sobre o sistema mola-massa.

\section{Considerações Finais}

O objetivo desta pesquisa foi o de investigar a utilização do ambiente de modelagem computacional semiquantitativo com base na metáfora de ícones WLinkIt no estudo de um tópico de Física: o sistema mola-massa. O mapeamento dos aspectos aqui relatados e discutidos em relação ao processo de construção de modelos do referido sistema físico através da utilização deste ambiente de modelagem computacional é fundamental para o delineamento e desenvolvimento de estudos futuros abordando outros sistemas físicos de relevância científica e educacional para o ensino de Física. Estes estudos têm o objetivo de promover, na prática, a integração destes ambientes no cotidiano da sala de aula a partir de conteúdos curriculares específicos.

\section{Agradecimentos}

Às instituições abaixo, que financiaram, parcialmente, este trabalho de pesquisa: CNPq, CAPES e FACITEC/CMT/PMV - Fundo de Apoio a Ciência e Tecnologia do município de Vitória-ES.

\section{Referências Bibliográficas}

BLISS J. OGBORN J (1989) Tools for Exploratory Learning. A Research Programme. Journal of Computer Assisted Learning, 5:37-50.

BLISS, J.; MONK, M \& OGBORN, J. (1983) Qualitative Data Analysis for Educational Research: a guide of systemic networs. 1. ed., London: Croom Helm. 215 p.

BLISS, J. et. al. (1992) Reasoning Supported by Computational Tools. Computer Education. Vol.18. p1-9.
BOOHAN, R. (1994) Creating Worlds from Objects and Events. In Mellar, H.; Bliss, J.; Boohan, R.;Ogborn, J. \& Tompsett, C. (eds.) Learning With Artificial Worlds: Computer Based Modelling in the Curriculum, (p.145-57). London: The Falmer Press.

CAMILETTI, G. (2001) Modelagem Computacional Semiquantitativa no Estudo de Tópicos de Ciências: Um Estudo Exploratório com Estudantes Universitários. Vitória, ES, Curso de Pós-Graduação em Física da Universidade Federal do Espírito Santo. Dissertação de Mestrado em Pesquisa em Ensino de Física.

CAMILETTI, G. \& FERRACIOLI, L. (2001) A Utilização da Modelagem Computacional Quantitativa no Aprendizado Exploratório de Física. Caderno Catarinense de Ensino de Fúsica, 18(2): 214-28.

FERRACIOLI, L \& SAMPAIO, F. F. (2001) Informação, Ciência, Tecnologia \& Inovação Curricular em Cursos de Licenciatura. Revista Brasileira de Informática na Educação, 8(1): 77-85.

FERRACIOLI, L. (1994) Commonsense Reasoning About Processes: A Study of Ideas about Reversibility. Ph.D. Thesis. London: Institute of Education University of London.

FERRACIOLI, L. (1997) As Novas Tecnologias nos Centros de Ciências, nos Centros de Formação Profissional e na Formação de Professores. In: Atas do XII Simpósio Nacional de Ensino de Física. Belo Horizonte: Universidade Federal de Minas Gerais. 2731/Janeiro/1997. p. 127-33.

FERRACIOLI, L. (2000) A Integração de Ambientes Computacionais ao Aprendizado Exploratório em Ciências. Projeto de Pesquisa CNPq, Processo $\mathrm{N}^{o} \_46.8522 / 00-0$.

FERRACIOLI, L. (2001) Aprendizagem, Desenvolvimento e Conhecimento na Obra de Jean Piaget: Uma Análise do Processo de Ensino-Aprendizagem em Ciências. Revista Brasileira de Estudos Pedagógicos. 80 (194): 5-18.

FORRESTER, J. (1968) Principles of Systems. Cambridge, Ma: Wright-Allen Press.

MANDINACH, E. B (1989) Model-Building and the Use of a Computer Simulation of Dynamics Systems. Journal Educational Computing Research, $\underline{5}$, (2): 221243.

OGBORN, J. \& MILLER, R. (1994) Computational Issues in Modelling. In Mellar, H.; Bliss, J.; Boohan, R.;Ogborn, J. \& Tompsett, C. (eds.) Learning With 
Artificial Worlds: Computer Based Modelling in the Curriculum, (p.117-27). London: The Falmer Press.

OGBORN, J. (1992) Modelling with the Computer at all ages. Publicação Interna do Institute of Education University of London.

OGBORN, J. (1994) The Nature of Modelling. In Mellar, H.; Bliss, J.; Boohan, R.; Ogborn, J. \& Tompsett, C. (eds.) Learning With Artificial Worlds: Computer Based Modelling in the Curriculum, (p.11-15). London: The Falmer Press

ROBERTS, N.; ANDERSEN, D.; DEAL, R.; GARET, M. \& SHAFFER, W. (1983) Introduction to Computer Simulation - A System Dynamic Modelling Approach. New York: Addison Wesley.

SAMPAIO, F. F. (1996) LinkIt: Design, Development and Testing of a Semi-Quantitative Computer Modelling Tool. Ph.D. Thesis, Department of Science and
Technology, Institute of Education, University of London.

SAMPAIO, F. F. TORRES, A. S. (1999) Trabalhando o conhecimento qualitativo de taxa de variação num ambiente de modelagem dinâmica computacional. III COINFE - Congresso Estadual de Informática na Educação. Instituto de Educação. Rio de Janeiro.

SANTOS A.C.K.; SAMPAIO, F.F. \& FERRACIOLI, L. (2001) Um Experimento de Modelagem Dinâmica Semiquantitativa com a Utilização da Técnica dos Hexágonos. Revista Brasileira de Informática na Educação, 7(1): 21-35.

SANTOS, A.C.K. \& OGBORN, J (1994) Sixth form students' ability to engage in computactional modelling. Journal of Computer Assisted Learning. 10:182200 . 